\title{
Stability of an inflated hyperelastic membrane tube with localized wall thinning *
}

\author{
A. T. Il'ichev ${ }^{1,2}$ and Y.B. Fu $\mathrm{u}^{3,4}$ \\ ${ }^{1}$ Steklov Mathematical Institute, Gubkina str. 8, 119991 Moscow, Russia \\ ${ }^{2}$ Bauman Moscow Technical University, Baumanskaya str. 5, 105110 Moscow, Russia \\ ${ }^{3}$ Department of Mathematics, Keele University, ST5 5BG, U.K. \\ ${ }^{4}$ Department of Mechanics, Tianjin University, Tianjin 300072, China
}

\begin{abstract}
It is now well-known that when an infinitely long hyperelastic membrane tube free from any imperfections is inflated, a transcritical-type bifurcation may take place that corresponds to the sudden formation of a localized bulge. When the membrane tube is subjected to localized wall-thinning, the bifurcation curve would "unfold" into the turning-point type with the lower branch corresponding to uniform inflation in the absence of imperfections, and the upper branch to bifurcated states with larger amplitude. In this paper stability of bulged configurations corresponding to both branches is investigated with the use of the spectral method. It is shown that under pressure control and with respect to axi-symmetric perturbations, configurations corresponding to the lower branch are stable but those corresponding to the upper branch are unstable. Stability or instability is established by demonstrating the non-existence or existence of an unstable eigenvalue (an eigenvalue with a positive real part). This is achieved by constructing the Evans function that depends only on the spectral parameter. This function is analytic in the right half of the complex plane where its zeroes correspond to the unstable eigenvalues of the generalized spectral problem governing spectral instability. We show that due to the fact that the skew-symmetric operator $\mathcal{J}$ involved in the Hamiltonian formulation of the basic equations is onto, the zeroes of the Evans function can only be located on the real axis of the complex plane. We also comment on the connection between spectral (linear) stability and nonlinear (Lyapunov) stability.
\end{abstract}

Keywords: Membrane tube; bifurcation; stability; nonlinear elasticity

*Dedicated to Professor Leonid M. Zubov on the occasion of his 70th birthday 


\section{Introduction}

This study is part of our recent research effort that explores the postulate that initiation of at least some acute aneurysms in human arteries may be modeled as a bifurcation phenomenon [8]. This postulate is motivated by two main results. Firstly, assuming that the artery is axisymmetric and homogeneous, and that the initial wall thickness is a constant, a localized bulge may form when the internal pressure reaches a certain critical value even if the pressure versus volume curve does not have a maximum in uniform inflation [7]. Secondly, when imperfections such as localized wall weakening is introduced, the bifurcation pressure may fall to within the physiologically possible range [10]. It is further postulated that it is after the initiation of such a localized bulge that biological processes such as remodelling take over, which in turn leads to further growth and final rupture of the aneurysm. The present study is also closely related to studies of solitary waves in hyperelastic membrane tubes; for a review of the relevant literature we refer to [5]. On the one hand, a static localized bulge can be viewed as a solitary wave that has zero propagation speed, the zero speed being induced by the internal pressure in the membrane tube. On the other hand, solitary waves, and more generally nonlinear waves, may play an important role in interrogating the health status of arteries (e.g. presence of an aneurysm) through signal processing [15], [17].

Identifying an aneurysm with a static localized bulge implies that this configuration must be stable since otherwise it cannot be observed. It was found that in the homogeneous case although internal fluid inertia would reduce the growth rate of the single unstable mode significantly, it alone cannot stabilize the unstable mode completely [13]. Stabilization of the exponential growth of the aneurysm solution takes place in the presence of a non-zero mean flow [6], but due to the translational symmetry of the problem the standing bulging configuration is only orbitally stable or stable in form [11]; it can still propagate axially with a non-zero speed under perturbations.

When wall weakening is introduced, the problem in question is no longer invariant under translations, and in this case we may speak about the usual stability of the standing configuration. In this paper we investigate the stability of bulging configurations corresponding to both branches of the bifurcation curve. In the absence of any imperfections, the lower branch would correspond to uniform inflation whose stability/bifurcation has previously been studied by Shield [18], Haughton \& Ogden [12], Chen [2], and Zubov and Sheidakov [19], and the upper branch would correspond to large amplitude bifurcated solutions whose stability properties have recently been studied by $\mathrm{Fu}$ and Xie [9]. Our stability analysis is based on the construction of the Evans function that depends only on the spectral parameter. The function is analytic in the right half of the complex plane and has there zeroes coinciding with unstable eigenvalues. We demonstrate that the zeroes of the Evans function can only be located on the real axis of the complex plane. Therefore, we need only to establish behavior of this function on the real axis which is technically possible, and based on this behavior we 
may draw conclusions not only about spectral instability of the bulging configurations under consideration, but also about its stability. In other words, absence of zeroes of the Evans function on the positive real axis implies linear stability of the aneurysm solution. Moreover, the correspondence of the spectrum of the related spectral problem in linear stability analysis to the one in Lyapunov (nonlinear) stability analysis is established. The spectrum $\eta$ of the linearized problem is related to the spectrum $-\alpha$ of the Hessian of the energy via the relation $\alpha=\rho \eta^{2}$, where $\rho$ is the density of the tube material. Therefore, with the Hessian being a self-adjoint operator, $\eta$ can only have real or purely imaginary values, the latter corresponding to the continuous spectrum. Linear instability is governed by the presence of a discrete spectrum.

The rest of the paper is divided into four sections as follows. After presenting the Hamiltonian form of the governing equations we discuss in Section 3 the construction of fully nonlinear bulging (aneurysm) solutions. We present the bifurcation diagram, reflecting the appearance of standing bulging solutions, and also a set of three first-order differential equations to be solved numerically to obtain the fully nonlinear bulging solutions. This is then followed by Section 4 where we discuss properties of the related spectral problem in the linear stability analysis. We construct the Evans function for both branches of the bifurcation diagram and examine its behavior on the real axis of the right half of the complex plane. According to the existence or absence of its zeroes conclusions about linear instability or stability of the aneurysm solutions in question are made. The paper is concluded in Section 5 with a brief discussion of the connections between linear spectral stability and nonlinear Lyapunov stability, and relevance of our results to the mathematical modelling of aneurysm initiation in human arteries.

\section{Formulation}

We consider the inflation of a cylindrical membrane tube that is assumed to be incompressible, isotropic, and hyperelastic. In its undeformed configuration, the tube wall has thickness $H$ that is not necessarily a constant, but the average of its outer and inner radii, hereafter referred to simply as the radius $R$, is a constant. The tube is assumed to be infinitely long, and end conditions are imposed at infinity. We use cylindrical polar coordinates, and undeformed and deformed configurations are described by coordinates $(R, \Theta, Z)$ and $(r, \theta, z)$, respectively.

We assume that the axisymmetry is maintained throughout the entire deformation, and so the deformation has the general form $r=r(Z, t), \theta=\Theta, z=z(Z, t)$. The principal directions of the deformation correspond to the lines of latitude, the meridian and the normal to the deformed surface, and the principal stretches are given by

$$
\lambda_{1}=\frac{r}{R}, \quad \lambda_{2}=\left(r^{\prime 2}+z^{\prime 2}\right)^{\frac{1}{2}}, \quad \lambda_{3}=\frac{h}{H}
$$


where a prime represents differentiation with respect to $Z$, and $h$ denotes the deformed thickness.

The principal Cauchy stresses $\sigma_{1}, \sigma_{2}, \sigma_{3}$ in the deformed configuration for an incompressible material are given by

$$
\sigma_{i}=\lambda_{i} \hat{W}_{i}-p, \quad i=1,2,3 \quad \text { (no summation) }
$$

where $\hat{W}=\hat{W}\left(\lambda_{1}, \lambda_{2}, \lambda_{3}\right)$ is the strain-energy function, $\hat{W}_{i}=\partial \hat{W} / \partial \lambda_{i}$, and $p$ is the pressure associated with the constraint of incompressibility. Utilizing the incompressibility constraint $\lambda_{1} \lambda_{2} \lambda_{3}=1$ and the membrane assumption of no stress through the thickness direction (i.e. $\sigma_{3}=0$ ), we find

$$
\sigma_{i}=\lambda_{i} W_{i}, \quad i=1,2 \quad \text { (no summation) }
$$

where $W\left(\lambda_{1}, \lambda_{2}\right)=\hat{W}\left(\lambda_{1}, \lambda_{2}, \lambda_{1}^{-1} \lambda_{2}^{-1}\right)$ and $W_{1}=\partial W / \partial \lambda_{1}$ etc. [12].

In our numerical illustrations, we shall assume that the membrane material is described by the Ogden strain-energy function

$$
\hat{W}=\sum_{r=1}^{3} \mu_{r}\left(\lambda_{1}^{\alpha_{r}}+\lambda_{2}^{\alpha_{r}}+\lambda_{3}^{\alpha_{r}}-3\right) / \alpha_{r}
$$

where

$$
\alpha_{1}=1.3, \quad \alpha_{2}=5.0, \quad \alpha_{3}=-2.0, \quad \mu_{1}=1.491, \quad \mu_{2}=0.003, \quad \mu_{3}=-0.023
$$

are material constants given by Ogden [16], and the $\mu$ 's have been scaled by the ground state shear modulus.

We consider the pressure controlled case when the inner pressure $P$ is prescribed. The total energy in the current configuration is $E=K+\Pi$, where $K$ is the kinetic energy given by

$$
K=\frac{1}{2} \int_{-L}^{L} \rho\left(\dot{r}^{2}+\dot{z}^{2}\right) 2 \pi R H d Z
$$

and $\Pi$ is the potential energy which is the sum of the strain energy and the potential energy of pressure:

$$
\Pi=\int_{-L}^{L} W\left(\lambda_{1}, \lambda_{2}\right) 2 \pi R H d Z-P \int_{-L}^{L} \pi r^{2} z^{\prime} d Z
$$

In the above expressions the superimposed dot denotes differentiation with respect to time and $L$ is the length of the tube in the undeformed configuration (which will shortly be taken to be infinite). The Hamiltonian, therefore, has the form

$$
E\left(q_{1}, q_{2}, v_{1}, v_{2}\right)=\frac{1}{2} \int_{-\infty}^{\infty}\left\{\rho R\left(v_{1}^{2}+v_{2}^{2}\right)+2 R\left(W\left(\lambda_{1}, \lambda_{2}\right)-W^{(\infty)}\right) H-P\left[\frac{q_{1}^{2}}{H}\left(\frac{q_{2}}{\sqrt{H}}\right)^{\prime}-r_{\infty}^{2} z_{\infty}\right]\right\} d Z
$$


where $q_{1}=\sqrt{H} r, q_{2}=\sqrt{H} z, v_{1}=\sqrt{H} \dot{r}, v_{2}=\sqrt{H} \dot{z}, W^{(\infty)}$ is the value of the strain-energy function $W$ at infinity where $\lambda_{1}=r_{\infty}, \lambda_{2}=z_{\infty}$. Here the constants are chosen such that the integral in (2.5) is convergent for $\left(r-r_{\infty}, z^{\prime}\right)$ exponentially decaying at infinity.

Employing $R$ as the unit of length, we may put $R$ in (2.5) to unity throughout this paper. If we denote $\boldsymbol{u}=\left\{q_{1}, q_{2}, v_{1}, v_{2}\right\}^{T}$, the equations of motion in the pressure controlled case may be written formally as a Hamiltonian dynamical system

$$
\frac{d \boldsymbol{u}}{d t}=\mathcal{J} \frac{\delta E}{\delta \boldsymbol{u}}, \quad \text { with } \quad \mathcal{J}=\frac{1}{\rho}\left(\begin{array}{cc}
0 & I \\
-I & 0
\end{array}\right),
$$

where $\delta / \delta \boldsymbol{u}$ denotes variational derivative and $I$ the $2 \times 2$ identity matrix. It can easily be verified that equation (2.6) is equivalent to the more familiar form [4]

$$
\begin{aligned}
& \rho \ddot{r}=\frac{1}{H}\left(\frac{H W_{2} r^{\prime}}{\lambda_{2}}\right)^{\prime}+\frac{P}{H} r z^{\prime}-W_{1}, \\
& \rho \ddot{z}=\frac{1}{H}\left(\frac{H W_{2} z^{\prime}}{\lambda_{2}}\right)^{\prime}-\frac{P}{H} r r^{\prime} .
\end{aligned}
$$

Taking the limit $Z \rightarrow \infty$ in $(2.7)_{1}$, we obtain

$$
P=\frac{H^{(\infty)} W_{1}^{(\infty)}}{r_{\infty} z_{\infty}}
$$

where superscript $(\infty)$ denotes evaluation at $Z=\infty$. We shall focus on the situation of an open-end membrane tube with fixed axial stretch $z_{\infty}$; this models the state of arteries. The azimuthal stretch $r_{\infty}$ can then be used as the control parameter in our bifurcation analysis, with the associated pressure calculated according to (2.8).

\section{Weakly and fully nonlinear bulging solutions}

The weakly nonlinear localized bulging solution has an amplitude of order $\epsilon$, where $\epsilon$ is a small enough positive dimensionless quantity. As in [10] we assume that the variable thickness $H$ has the form

$$
H=H^{(\infty)}\left(1+\epsilon^{2} a(\xi)\right), \quad \xi=\epsilon Z, \quad a( \pm \infty) \rightarrow 0,
$$

where $H^{(\infty)}$ is the constant wall thickness at infinity and the function $a(\xi)$ is to be prescribed.

It was shown in [10] that if $r-r_{\infty}=\epsilon y(\xi)$ for weakly nonlinear solutions, $y$ must satisfy the differential equation

$$
\frac{d^{2} y}{d \xi^{2}}=\omega_{c r}^{\prime} r_{1} y+\frac{3}{2} \gamma_{c r} y^{2}+\zeta a(\xi)
$$

where $r_{1}$ is defined by $r_{\infty}=r_{c r}+\epsilon r_{1}$, with $r_{c r}$ being the critical value of $r_{\infty}$ at which a bulge will initiate without any imperfections, $\omega_{c r}^{\prime}=d \omega\left(r_{c r}\right) / d r_{c r}, \gamma_{c r}=\gamma\left(r_{c r}\right)$, and explicit 
expressions for $\omega\left(r_{\infty}\right), \gamma\left(r_{\infty}\right)$ and $\zeta$ in terms of the strain-energy function can be found in [7], [5] and [10], respectively. In [10] several classes of $a(\xi)$ are considered for which (3.1) has closed-form solutions. In particular, if $a(\xi)$ takes the form

$$
a(\xi)=\frac{3}{2} d_{1} y^{2}
$$

where $d_{1}$ is a constant, then (3.1) has an explicit localized solution given by

$$
y=-\frac{\omega_{c r}^{\prime} r_{1}}{\gamma_{c r}+\zeta d_{1}} \operatorname{sech}^{2}\left(\frac{1}{2} \sqrt{\omega_{c r}^{\prime} r_{1}} \xi\right)
$$

Denoting $a(0)$ by $a_{0}$, we have

$$
a_{0}=\frac{3}{2} d_{1} y^{2}(0)=\frac{3 d_{1}\left(\omega_{c r}^{\prime} r_{1}\right)^{2}}{2\left(\gamma_{c r}+\zeta d_{1}\right)^{2}}
$$

which can be solved to express $d_{1}$, and hence $a(\xi)$ and $y(\xi)$, in terms of $a_{0}$. We then obtain

$$
r_{0}-r_{\infty}=\epsilon y(0)=-\frac{\omega_{c r}^{\prime} \cdot\left(r_{\infty}-r_{c r}\right)}{2 \gamma_{c r}}\left[1 \pm \sqrt{1-\frac{8 \gamma_{c r} a_{0} \zeta \epsilon^{2}}{3 \omega_{c r}^{\prime 2}\left(r_{\infty}-r_{c r}\right)^{2}}}\right] .
$$

Plotted on the $\left(r_{\infty}, r_{0}-r_{\infty}\right)$-plane, the above expression describes a parabola opening to the left when $\gamma_{c r} a_{0} \zeta>0$. The turning point (i.e. the nose of the parabola), beyond which no localized solutions can exist, corresponds to

$$
r_{\infty}=r_{c r}+2 \sqrt{\frac{2}{3}} \cdot \frac{\epsilon \sqrt{\gamma_{c r} \zeta a_{0}}}{\omega_{c r}^{\prime}}
$$

For the Ogden material model and in the open end case with $z_{\infty}=1$, we have

$$
\zeta=2.0328, r_{c r}=1.6873, \omega_{c r}^{\prime}=-3.2329, \gamma_{c r}=-1.3369 .
$$

Thus, the expression (3.5) is real only for the wall-thinning case (i.e. $a_{0}<0$ ), and its right hand side is less than $r_{c r}$ by an amount that is proportional to the square root of the imperfection amplitude $H_{\infty}-H(0)$. This reflects the square root law for the imperfection sensitivity of this type of elastic localizations [10].

Although the particular choice of the wall thinning profile (3.2) leads to an exact solution that enables us to see explicitly how the bifurcation diagram unfolds from the perfect case, this profile is actually dependent on $r_{1}$ and hence on the value of $r_{\infty}$. In our subsequent calculations, we shall consider the $r_{\infty}$-independent profile

$$
H(Z)=H^{(\infty)}\left(1-0.05 \operatorname{sech}^{4} Z\right) .
$$

The associated bifurcation diagram is obtained as follows. First, the equilibrium equations can be obtained from (2.7) and may be written in the form (see, e.g., [10])

$$
\begin{aligned}
\lambda_{1}^{\prime} & =\lambda_{2} \sin \phi \\
\lambda_{2}^{\prime} & =\frac{W_{1}-\lambda_{2} W_{12}}{W_{22}} \sin \phi-\frac{H^{\prime} W_{2}}{W_{22}}, \\
\phi^{\prime} & =\frac{W_{1}}{W_{2}}-\frac{P \lambda_{1} \lambda_{2}}{H W_{2}}
\end{aligned}
$$


where $\phi$ is the angle between the meridian and the $Z$-axis so that $r^{\prime}=\lambda_{2} \sin \phi, z^{\prime}=\lambda_{2} \cos \phi$. We also note that $(2.7)_{2}$ in the static case can be integrated once to give

$$
\frac{H W_{2} z^{\prime}}{\lambda_{2}}-\frac{1}{2} \operatorname{Pr}^{2}=C_{1}
$$

where the integration constant $C_{1}$ can be determined by evaluating the left hand side at $\infty$. The (symmetric) localized bulging solutions can then be determined by integrating the system (3.7) from $Z=0$ towards $\infty$ subject to the initial conditions

$$
\lambda_{1}(0)=r_{0}, \quad \lambda_{2}(0)=z_{0}^{\prime}, \quad \phi(0)=0,
$$

where $r_{0}$ is to be guessed in our shooting procedure, and the constant $z_{0}^{\prime}$ is related to $r_{0}$ by

$$
f\left(r_{0}, z_{0}^{\prime}\right) \equiv H(0) W_{2}\left(r_{0}, z_{0}^{\prime}\right)-H^{(\infty)} W^{(\infty)}-\frac{1}{2} P\left(r_{0}^{2}-r_{\infty}^{2}\right)=0
$$

obtained from the integral (3.8). The solvability of (3.9) for $z_{0}^{\prime}$ is guaranteed by the fact that $\partial f / \partial z_{0}^{\prime}=H(0) W_{22}\left(r_{0}, z_{0}^{\prime}\right)>0$, whose satisfaction is verified numerically (this can also be made as a constitutive assumption, see, e.g., [12]). Thus, for each specified $r_{\infty}$ and a guess for $r_{0}$, we solve (3.9) numerically to find the corresponding $z_{0}^{\prime}$. We iterate on $r_{0}$ so that the decay condition $[10]$

$$
r^{\prime}(L)+\sqrt{\omega\left(r_{\infty}\right)}\left(r(L)-r_{\infty}\right)=0
$$

is satisfied for a sufficiently large positive number $L$. In Figure 1, we have shown the dependence of $r(0)-r_{\infty}$ on $r_{\infty}$ corresponding to the wall thickness profile (3.6). It is seen that it has a similar form to the one described by the analytical expression (3.5). We also observe that the upper branch very quickly approaches its counterpart in the absence of imperfections. This means that large amplitude bulged solutions do not feel the presence of the initial wall-thinning, the main effect of the latter being to reduce the bifurcation value of $r_{\infty}$.

\section{$4 \quad$ Stability analysis}

Denoting the static bulging (aneurysm) solutions obtained in the previous section by $\bar{r}(Z)$ and $\bar{z}(Z)$, we now consider its linear stability subject to axisymmetric perturbations. Defin$\operatorname{ing} \bar{q}_{1}(Z)=\sqrt{H} \bar{r}, \bar{q}_{2}(Z)=\sqrt{H} \bar{z}(Z)$, we write

$$
q_{1}(Z, t)=\bar{q}_{1}(Z)+\Psi(Z) \mathrm{e}^{\eta t}, \quad q_{2}(Z, t)=\bar{q}_{2}(Z)+\Phi(Z) \mathrm{e}^{\eta t}
$$

where the mode functions $\Psi(Z), \Phi(Z)$ and the growth rate $\eta$ are to be determined. On substituting expressions (4.1) into (2.6), or equivalently (2.7), and linearizing, we find

$$
\mathcal{L} \boldsymbol{B}=\rho \eta^{2} \boldsymbol{B}, \quad \text { with } \quad \boldsymbol{B}=\{\Psi, \Phi\}^{T},
$$




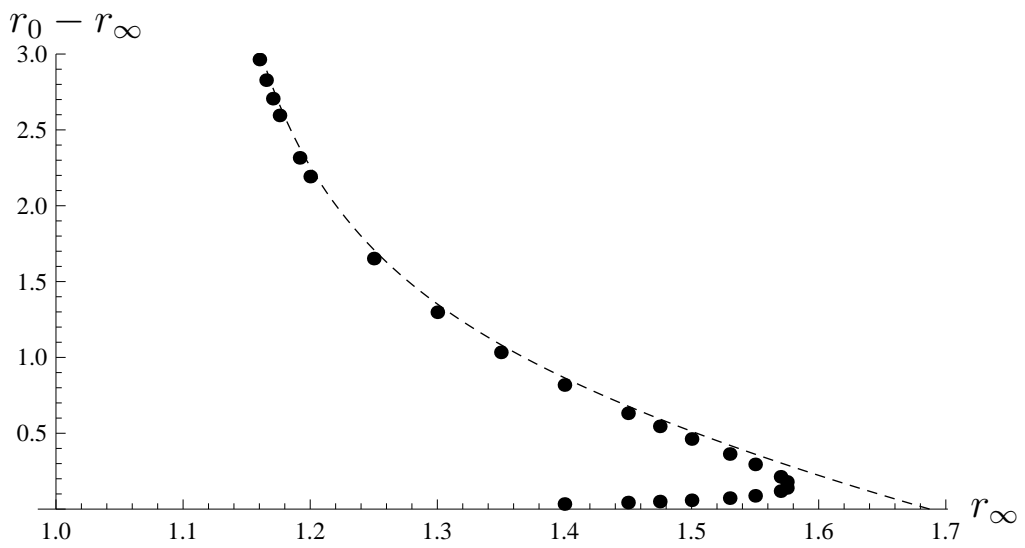

Figure 1: Dependence of $r(0)-r_{\infty}$ on $r_{\infty}$ when the wall thickness is given by (3.6) (dark dots). The corresponding result when the wall thickness is uniform is given by the dashed line. The main effect of localized wall thinning is to reduce the bifurcation value of $r_{\infty}$ from 1.687 to 1.576 (a $6.6 \%$ reduction); it has a negligible effect on large amplitude bulged solutions.

where the differential operator $\mathcal{L}$ is not written out for brevity.

As the aneurysm solution is a stationary solution, we have

$$
D E(\overline{\boldsymbol{u}}) \equiv \frac{\delta}{\delta \boldsymbol{u}} E(\overline{\boldsymbol{u}})=0
$$

where $\overline{\boldsymbol{u}}=\left\{\overline{q_{1}}, \overline{q_{2}}, 0,0\right\}^{T}$. The Hessian of $E$ evaluated at the aneurysm solution is

$$
\mathcal{H}=D^{2} E(\overline{\boldsymbol{u}})=\left(\begin{array}{cc}
-\mathcal{L} & 0 \\
0 & \rho I
\end{array}\right),
$$

where $I$ is the $2 \times 2$ identity matrix and $\mathcal{L}$ is the same operator as in (4.2). It is shown in [11] that the operator $\mathcal{H}$ constructed in this way is self-adjoint. It then follows that $\mathcal{L}$ must necessarily be a self-adjoint operator and so it can have only real spectrum. Thus, the eigenvalues (discrete spectrum) $\rho \eta^{2}$ in (4.2) can only lie on the real axis, that is, $\eta$ can only be real or pure imaginary.

It is seen that Eq. (4.2) is a system of two coupled linear non-autonomous second order differential equations, and the dependence on $\eta$ is entirely through $\eta^{2}$. We denote $\alpha=\rho \eta^{2}$. Eq. (4.2) can then be written in the form

$$
\boldsymbol{y}^{\prime}=\mathcal{M} \boldsymbol{y}
$$

where $\boldsymbol{y}=\left(\Psi, \Psi^{\prime}, \Phi, \Phi^{\prime}\right)^{T}$ and $\mathcal{M}$ is a $4 \times 4$ matrix whose components are not written out for brevity. Eq. (4.4) subject to the decay conditions $\boldsymbol{y} \rightarrow \mathbf{0}$ as $Z \rightarrow \pm \infty$ is an eigenvalue problem for $\alpha$. The aneurysm solution is said to be unstable if this eigenvalue problem has a positive eigenvalue.

We denote by $\mathcal{M}_{\infty}$ the limit of $\mathcal{M}$ as $Z \rightarrow \pm \infty$. Eq. (4.4) then asymptotes to a constant coefficient problem with exponential solutions $\exp (k Z)$, for values of $k$ related 
to the parameter $\alpha$ by the equation

$$
\operatorname{det}\left(\mathcal{M}_{\infty}-k I\right)=0
$$

where $I$ is the $4 \times 4$ identity matrix. On evaluating the determinant, we obtain

$$
\alpha_{0} \gamma_{1} \hat{k}^{4}+\left[\left(\alpha_{1}-\beta_{0}\right)^{2}-\left(\beta_{1}-\beta_{0}\right) \gamma_{1}-\hat{c}^{2}\left(\alpha_{0}+\gamma_{1}\right)\right] \hat{k}^{2}+\left(\hat{c}^{2}+\beta_{1}-\beta_{0}\right) \hat{c}^{2}=0,
$$

where

$$
\hat{c}^{2}=\alpha r_{\infty}^{2}=\rho \eta^{2} r_{\infty}^{2}, \quad \hat{k}=\frac{r_{\infty}}{z_{\infty}} k,
$$

and the material constants $\alpha_{0}, \alpha_{1}, \beta_{0}, \beta_{1}, \gamma_{1}$ are defined in [5].

It can easily be seen that the four eigenvalues of $\mathcal{M}_{\infty}$ take the form $\pm k_{1}, \pm k_{2}$. It can be proved by an argument similar to that in [13] that $\hat{k}$ can be pure imaginary if and only if $\hat{c}$ is pure imaginary (or equivalently, if $\hat{c}^{2}$ is real and negative). Therefore, for our construction of unstable eigenfunctions (positive $\hat{c}^{2}$ ) the roots $\hat{k_{1}}$ and $\hat{k_{2}}$ cannot cross the imaginary axis of the complex plane, and the four eigenvalues are symmetric with respect to both the real and imaginary axes. Without loss of generality, we assume that it is the $k_{1}$ and $k_{2}$ that have a negative real part. The system of equations (4.4) then has two independent solutions, $\boldsymbol{y}_{1}$ and $\boldsymbol{y}_{2}$ say, that decay as $Z \rightarrow \infty$ like $\mathrm{e}^{k_{1} Z}$ and $\mathrm{e}^{k_{2} Z}$, respectively, and another two independent solutions, $\boldsymbol{y}_{1}^{-}$and $\boldsymbol{y}_{2}^{-}$say, that decay as $Z \rightarrow-\infty$ like $\mathrm{e}^{-k_{1} Z}$ and $\mathrm{e}^{-k_{2} Z}$, respectively.

We also observe in passing that each pair of pure imaginary $\hat{k}$ and $\hat{c}$ corresponds to one of the four possible traveling waves that may propagate in the pressurized tube. It can be seen from (4.5) that two branches of the dispersion curve has the behaviour $|\hat{c}| \rightarrow 0$ as $\hat{k} \rightarrow 0$. It thus follows that the continuous spectrum of $\mathcal{L}$ is given by $(-\infty, 0)$.

The eigenvalue problem (4.4) can be solved in a number of ways. The most straightforward approach is the so-called determinant method, which determines $\alpha$ by solving the equation

$$
\operatorname{det}\left(\boldsymbol{y}_{1}, \boldsymbol{y}_{2}, \boldsymbol{y}_{1}^{-}, \boldsymbol{y}_{2}^{-}\right)=0,
$$

where the left hand side can be evaluated at any appropriate matching point on the real line. The method suffers from the "stiffness" problem in the sense that one column can get dominated by another column due to different exponential behaviour. A better method is the compound matrix method. One version of this method is used in [14]. In this paper we employ another version of this method which is usually called the Evans function method in the nonlinear waves community.

To solve the eigenvalue problem (4.4) using the Evans function method, we first define the following adjoint of (4.4):

$$
\boldsymbol{x}^{\prime}=-\mathcal{M}^{T} \boldsymbol{x}
$$

For each $\boldsymbol{y}(\alpha, Z)$ and $\boldsymbol{x}(\alpha, Z)$ that satisfy (4.4), (4.7), respectively, it can easily be verified that

$$
\frac{\partial}{\partial Z}(\boldsymbol{x}(\alpha, Z) \cdot \boldsymbol{y}(\alpha, Z))=0
$$


where "." denotes the usual scalar product between two vectors. Thus, if both $\boldsymbol{x}$ and $\boldsymbol{y}$ in (4.8) are bounded and at least one of them decays to zero as $Z \rightarrow \infty$ or $-\infty$, then their dot product is identically zero. This property will be used shortly in the analysis below.

Denote by $\boldsymbol{a}_{1}, \boldsymbol{a}_{2}$ the right eigenvectors of $\mathcal{M}_{\infty}$ associated with the eigenvalues $k_{1}$ and $k_{2}$, and by $\boldsymbol{b}_{1}, \boldsymbol{b}_{2}$ the left eigenvectors of $\mathcal{M}_{\infty}$ associated with the eigenvalues $-k_{1}$ and $-k_{2}$. From the general theory of ordinary differential equations it follows (see [3]) that there exist the solutions $\boldsymbol{y}_{1}(\alpha, Z), \boldsymbol{y}_{2}(\alpha, Z), \boldsymbol{x}_{1}(\alpha, Z)$, and $\boldsymbol{x}_{2}(\alpha, Z)$ of (4.4) and (4.7), such that

$$
\lim _{Z \rightarrow \infty} \mathrm{e}^{-k_{i} Z} \boldsymbol{y}_{i}(\alpha, Z)=\boldsymbol{a}_{k}(\lambda), \quad \lim _{Z \rightarrow-\infty} \mathrm{e}^{k_{i} Z} \boldsymbol{x}_{i}(\alpha, Z)=\boldsymbol{b}_{k}(\lambda), \quad i=1,2 .
$$

A general solution that decays exponentially as $Z \rightarrow \infty$ is given by

$$
\boldsymbol{y}=c_{1} \boldsymbol{y}_{1}(\alpha, Z)+c_{2} \boldsymbol{y}_{2}(\alpha, Z)=\left(\boldsymbol{y}_{1}, \boldsymbol{y}_{2}\right)\left(\begin{array}{c}
c_{1} \\
c_{2}
\end{array}\right)
$$

where $c_{1}$ and $c_{2}$ are constants. It follows from (4.8) and the decay behaviour of $\boldsymbol{y}$ that

$$
\mathbf{0}=\left(\begin{array}{c}
\boldsymbol{x}_{1} \cdot \boldsymbol{y} \\
\boldsymbol{x}_{2} \cdot \boldsymbol{y}
\end{array}\right)=\left(\begin{array}{c}
\boldsymbol{x}_{1}^{T} \\
\boldsymbol{x}_{2}^{T}
\end{array}\right)\left(\boldsymbol{y}_{1}, \boldsymbol{y}_{2}\right)\left(\begin{array}{c}
c_{1} \\
c_{2}
\end{array}\right),
$$

and so for a non-trivial solution we must have

$$
\operatorname{det}\left(\begin{array}{c}
\boldsymbol{x}_{1}^{T} \\
\boldsymbol{x}_{2}^{T}
\end{array}\right)\left(\boldsymbol{y}_{1}, \boldsymbol{y}_{2}\right)=0 .
$$

This condition is equivalent to (4.6), and its direct numerical computation would suffer from the same stiffness problem. In the following, this determinant is evaluated with the aid of the associated exterior systems (or in terms of the compound matrices).

\subsection{Exterior systems}

Consider the vectors $\boldsymbol{y}^{\wedge}(\alpha, Z)$ and $\boldsymbol{x}^{\wedge}(\alpha, Z)$ with components defined by

$$
y_{\beta \wedge \gamma}^{\wedge}=y_{1 \beta} y_{2 \gamma}-y_{1 \gamma} y_{2 \beta}, \quad x_{\beta \wedge \gamma}^{\wedge}=x_{1 \beta} x_{2 \gamma}-x_{1 \gamma} x_{2 \beta}, \quad \beta<\gamma,
$$

where $\beta, \gamma=1,2,3,4$, and $y_{k \beta}$ and $x_{k \beta}$ are the $\beta$-th components of the vectors $\boldsymbol{y}_{k}$ and $\boldsymbol{x}_{k}$, respectively. We use the following correspondence between $\alpha \wedge \beta$ and the numbers 1 to 6 :

$$
1 \wedge 2 \rightarrow 1,1 \wedge 3 \rightarrow 2,1 \wedge 4 \rightarrow 3,2 \wedge 3 \rightarrow 4,2 \wedge 4 \rightarrow 5,3 \wedge 4 \rightarrow 6
$$

We observe that the two vectors $\boldsymbol{y}^{\wedge}$ and $\boldsymbol{x}^{\wedge}$ defined above are simply the (2nd) compound matrices of $\left(\boldsymbol{y}_{1}, \boldsymbol{y}_{2}\right)$ and $\left(\boldsymbol{x}_{1}, \boldsymbol{x}_{2}\right)$, respectively.

The vectors $\boldsymbol{y}^{\wedge}(\alpha, Z)$ and $\boldsymbol{x}^{\wedge}(\alpha, Z)$ satisfy the linear systems

$$
\frac{d}{d Z} \boldsymbol{y}^{\wedge}=\mathcal{M}^{\wedge}(\alpha, Z) \boldsymbol{y}^{\wedge}, \quad \frac{d}{d Z} \boldsymbol{x}^{\wedge}=-\left[\mathcal{M}^{\wedge}(\alpha, Z)\right]^{T} \boldsymbol{x}^{\wedge}
$$


We define the asymptotic matrix

$$
\mathcal{M}_{\infty}^{\wedge}(\alpha)=\lim _{Z \rightarrow \pm \infty} \mathcal{M}^{\wedge}(\alpha, Z)
$$

It is well-known that the six eigenvalues of $\mathcal{M}_{\infty}^{\wedge}(\alpha)$ are given by

$$
k_{\alpha}(\alpha)+k_{\beta}(\alpha), \quad 1 \leq \alpha<\beta \leq 4
$$

\subsection{Evans function}

For $\eta$ in the right complex half-plane, the matrix $\mathcal{M}_{\infty}(\alpha)$ has two eigenvalues $k_{1}(\alpha)$ and $k_{2}(\alpha)$ in the left half-plane. Thus the matrix $\mathcal{M}_{\infty}^{\wedge}(\alpha)$ has simple (hence analytic) left-most eigenvalue $k^{\wedge}(\alpha)=k_{1}(\alpha)+k_{2}(\alpha)$ for $\eta$ in the right half-plane. By exact analogy with (4.9), there are solutions of (4.14) such that

$$
\lim _{Z \rightarrow \infty} \mathrm{e}^{-k^{\wedge}(\alpha) Z} \boldsymbol{y}^{\wedge}(\alpha, Z)=\boldsymbol{a}^{\wedge}(\alpha), \quad \lim _{Z \rightarrow-\infty} \mathrm{e}^{k^{\wedge}(\alpha) Z} \boldsymbol{x}^{\wedge}(\alpha, Z)=\boldsymbol{b}^{\wedge}(\alpha)
$$

where $\boldsymbol{a}^{\wedge}(\alpha)$ is the right eigenvectors of $\mathcal{M}_{\infty}^{\wedge}(\alpha)$ associated with the eigenvalue $k^{\wedge}(\alpha)$, and $\boldsymbol{b}^{\wedge}(\alpha)$ is the left eigenvectors of $\mathcal{M}_{\infty}^{\wedge}(\alpha)$ associated with the eigenvalue $-k^{\wedge}(\alpha)$.

We define the Evans function by

$$
D(\alpha)=\boldsymbol{x}^{\wedge} \cdot \boldsymbol{y}^{\wedge}
$$

It is a standard result that $\boldsymbol{x}^{\wedge} \cdot \boldsymbol{y}^{\wedge}$ defined above is equal to the determinant on the left hand side of (4.12). Thus, the above construction is simply an alternative way to evaluate (4.12) that avoids any stiffness behaviour. Since the eigenvalue $k^{\wedge}(\eta)$ is simple, the argument of Alexander and Sachs [1] can be used to show that the Evans function defined by (4.15) is analytic in the entire complex right half-plane of $\eta$ and it is real for real $\eta$.

For $\operatorname{Re} \eta>0$ the function $D(\alpha)$ is zero if and only if there is a solution of (4.4) (i.e. an unstable eigenfunction) which decays exponentially as $Z \rightarrow \pm \infty$ (see, for example, [1]).

\subsection{Numerical results}

For the different bulging solutions represented by the bifurcation diagram in Figure 1, no unstable eigenvalues are found for any solution corresponding to the lower branch. For each solution corresponding to the upper branch, we found a single unstable eigenvalue. The dependence of $\alpha$ on $r_{\infty}$ is displayed in Figure 2. It is seen that the growth rate of the single unstable mode tends to zero in two limits. The right one corresponds to the turning point in Figure 1, whereas the left limit corresponds to the case when the bulging solution becomes a "hat" solution. The hat solution has the property that at its centre $r^{\prime \prime}(0)$ is zero as well as $r^{\prime}(0)$, and therefore, it can be viewed as two kink solutions joined together. The growth rate of the unstable mode tending to zero in the latter limit is consistent with the fact that the kink solution is usually observed to be stable in experiments. 


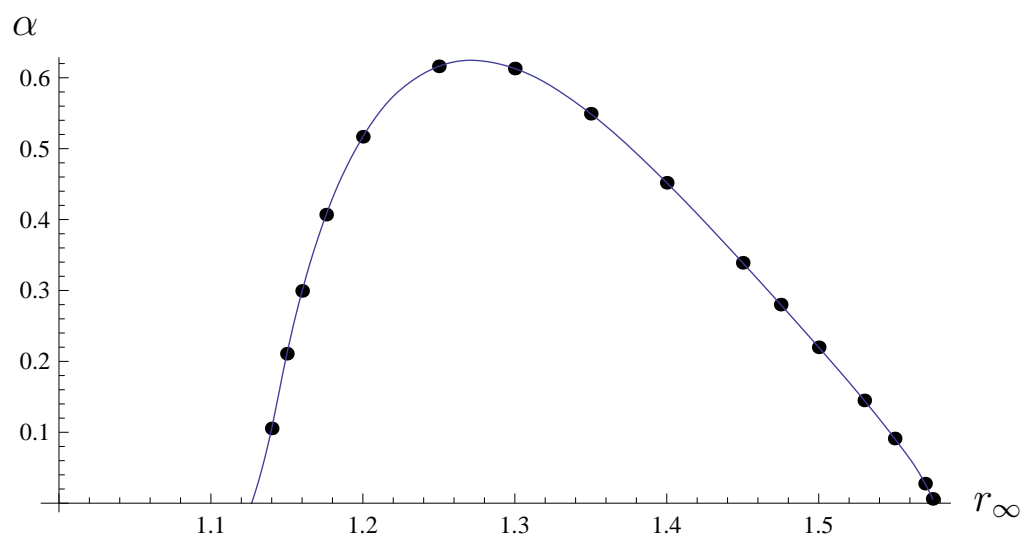

Figure 2: Dependence of the single eigenvalue of $\alpha=\rho \eta^{2}$ on $r_{\infty}$. The solid line is the quadratic spline interpolation of the finite set of numerical results represented by the dots.

\section{Conclusion}

Although we have so far focussed on a spectral stability analysis, we now show that there is in fact a connection between spectral (linear) stability and nonlinear (Lyapunov) stability.

Suppose that we superimpose a small-amplitude localized perturbation $\boldsymbol{v}(Z, t)$ on the static aneurysm solution $\overline{\boldsymbol{u}}(Z)$. We then have

$$
E(\overline{\boldsymbol{u}}+\boldsymbol{v})-E(\overline{\boldsymbol{u}})=\frac{1}{2}\langle\mathcal{H} \boldsymbol{v}, \boldsymbol{v}\rangle+o\left(\|\boldsymbol{v}\|^{2}\right),
$$

where the operator $\mathcal{H}$ is defined by (4.3) and $\|\boldsymbol{v}\|$ denotes the $L^{2}$-norm of $\boldsymbol{v}$ on $(-\infty, \infty)$. Thus, if $\mathcal{H}$ has only positive eigenvalues and if its continuous spectrum is bounded away from zero, then there exists a positive constant $c$ such that $\langle\mathcal{H} \boldsymbol{v}, \boldsymbol{v}\rangle \geq c\|\boldsymbol{v}\|^{2}$ (see, e.g., [11]). It then follows immediately that there exists another positive constant $c_{1}$ such that

$$
E(\overline{\boldsymbol{u}}+\boldsymbol{v}(Z, 0))-E(\overline{\boldsymbol{u}})=E(\overline{\boldsymbol{u}}+\boldsymbol{v}(Z, t))-E(\overline{\boldsymbol{u}}) \geq c_{1}\|\boldsymbol{v}\|^{2}
$$

where use has been made of the fact that the Hamiltonian is a conserved quantity. This estimate together with the continuity of $E$ would give us nonlinear (Lyapunov) stability since $E(\overline{\boldsymbol{u}}+\boldsymbol{v}(Z, 0))-E(\overline{\boldsymbol{u}})$, and hence $\|\boldsymbol{v}\|$, can be made arbitrarily small by choosing the initial perturbation $\boldsymbol{v}(Z, 0)$ to be sufficiently small.

The eigenvalues of $\mathcal{H}$ are computed according to $\mathcal{H} \boldsymbol{\chi}=-\alpha \boldsymbol{\chi}$, where $-\alpha$ is the spectral parameter. By comparing the top half of this matrix equation with (4.2), we obtain $\alpha=\rho \eta^{2}$. Thus, the $\alpha$ defined here has the same meaning as the one defined above (4.4). Suppose that the spectral problem discussed in the previous sections does not have a positive eigenvalue $\alpha$. Then the operator $\mathcal{H}$ does not have any negative eigenvalues. However, since the continuous spectrum of $\mathcal{H}$ is minus that of $\mathcal{L}$, it is given by $(0, \infty)$ and so is not separated from zero. Thus, we cannot deduce nonlinear (Lyapunov) stability. Nonetheless, in practice the tube cannot be infinitely long. For a finite tube, the positive continuous spectrum transforms 
into positive discrete eigenvalues, and the latter are the only eigenvalues of $\mathcal{H}$ since zero is not an eigenvalue due to the fact that the problem has no translational invariance in the presence of imperfections. Therefore, linear spectral stability of the bulge in question would practically imply nonlinear (Lyapunov) stability.

To conclude, we have shown in this paper that configurations corresponding to the upper branch of the bifurcation curve (see Fig. 1) is linearly unstable, but those corresponding to the lowest branch are stable. We note that towards the turning point on the bifurcation diagram, stable configurations on the lower branch may have a significant amplitude (compared with the case when no bifurcation is possible and bulging evolution is entirely due to initial imperfections). In the context of blood flow in an artery, such a large amplitude bulge would modify the flow properties, which in turn would provide a possible mechanism triggering arterial wall growth and remodeling. Based on our recent study on the effects of a mean flow [6], we envisage that a mean flow may stabilize configurations corresponding to the upper branch. If this were proved to be the case, stable bulged configurations with even larger amplitude would become possible and their effects on the blood flow would be even more pronounced. However, we appreciate that there is still a lot of uncertainty in the constitutive modeling of arterial walls in vivo and whether bifurcation is possible depends very much on the material models used [8]. Our results should at least be indicative of what might happen if bifurcation is possible when more realistic material models are used.

\section{Acknowledgements}

This work is supported by a Joint Project grant awarded by the Royal Society and Russian Foundation for Basic Science Research. The research of the first author (AI) is also supported by the Russian Foundation for Basic Research (Project No. 11-01-00034-a), and the research of the second author (YF) is also supported by the National Science Foundation of China (grant no. 11372212). The authors thank an anonymous referee for his/her helpful comments.

\section{References}

[1] J.C. Alexander, K. Sachs, Linear instability of solitary waves of a Boussinesq type equation: A computer assisted computation, Nonlin. World, 2 (1995) 471-507.

[2] Y.-C. Chen, Stability and bifurcation of finite deformations of elastic cylindrical membranes - part I. stability analysis, Int. J. Solids Structures 34 (1997) 1735-1749.

[3] E.A. Coddington, N. Levinson, Theory of ordinary differential equations, New York, McGraw Hill, 1955. 
[4] M. Epstein, C. Johnston, On the exact speed and amplitude of solitary waves in fluidfilled elastic tubes, Proc. R. Soc. Lond. A457 (2001) 1195-1213.

[5] Y.B. Fu, A. Il'ichev, Solitary waves in fluid-filled elastic tubes: existence, persistence, and the role of axial displacement, IMA J. Appl. Math. 75 (2010) 257-268.

[6] Y.B. Fu, A. Il'ichev, Localized standing waves in a hyperelastic membrane tube and their stabilization by a mean flow, Maths Mech. Solids, to appear.

[7] Y.B. Fu, S.P. Pearce, K.K. Liu, Post-bifurcation analysis of a thin-walled hyperelastic tube under inflation, Int. J. Non-linear Mech. 43 (2008) 697-706.

[8] Y.B. Fu, G.A. Rogerson, Y.T. Zhang, Initiation of aneurysms as a mechanical bifurcation phenomenon, Int. J. Non-linear Mech. 47 (2012) 179-184.

[9] Y.B. Fu, Y.X. Xie, Stability of localized bulging in inflated membrane tubes under volume control, Int. J. Eng. Sci. 48 (2010) 1242-1252.

[10] Y.B. Fu, Y.X. Xie, Effects of imperfections on localized bulging in inflated membrane tubes, Phil. Trans. R. Soc. A370 (2012) 1896-1911.

[11] M. Grillakis, J. Shatah, W. Strauss, Stability theory of solitary waves in the presence of symmetry. I, Journ. Funct. Anal. 74 (1987) 160-197.

[12] D.M. Haughton, R.W. Ogden, Bifurcation of inflated circular cylinders of elastic material under axial loading. I. Membrane theory for thin-walled tubes, J. Mech. Phys. Solids 27 (1979) 179-212.

[13] A.T. Ilichev, Y.B. Fu, Stability of aneurysm solutions in a fluid-filled elastic membrane tube, Acta Mechanica Sinica 28 (2012) 1209-1218.

[14] S.P. Pearce, Y.B. Fu, Characterisation and stability of localised bulging/necking in inflated membrane tubes, IMA J. Appl. Math. 75 (2010) 581-602.

[15] S. Noubissié, R.A. Kraenkel, P. Woafo, Disturbance and repair of solitary waves in blood vessels with aneeurysm. Commun. Nonlinear Sci. Numer. Simul. 14 (2009) 51-60.

[16] R.W. Ogden, Large deformation isotropic elasticity-on the correlation of theory and experiment for incompressible rubber-like solids. Proc. R. Soc. Lond. A326 (1972) 565584.

[17] K.H. Parker, An introduction to wave intensity analysis, Med. Biol. Eng. Comput. 47 (2009) 175-188.

[18] R.T. Shield, On the stability of finitely deformed elastic membranes; Part II: Stability of inflated cylindrical and spherical membranes, ZAMP 23 (1972) 16-34. 
[19] L.M. Zubov, D.N. Sheidakov, Instability of a hollow elastic cylinder under tension, torsion, and inflation, ASME J. Appl. Mech. 75 (2008) 011002. 